International Journal of Pure and Applied Mathematics

Volume 84 No. 1 2013, 115-139

ISSN: 1311-8080 (printed version); ISSN: 1314-3395 (on-line version)

url: http://www.ijpam.eu

doi: http://dx.doi.org/10.12732/ijpam.v84i1.9

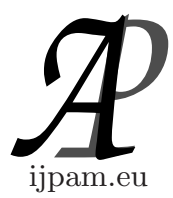

\title{
COMPUTING DOT - PRODUCT ON HETEROGENEOUS MASTER - WORKER PLATFORMS
}

\author{
Panagiotis D. Michailidis ${ }^{1}$, Konstantinos G. Margaritis ${ }^{2} \S$ \\ ${ }^{1}$ Department of Balkan Studies \\ University of Western Macedonia \\ 3rd Km Florinas-Nikis National Road, Florina, 53100, GREECE \\ ${ }^{2}$ Department of Applied Informatics \\ University of Macedonia
}

156 Egnatia str., Thessaloniki, 54006, GREECE

\begin{abstract}
This paper is focused on designing two parallel dot product implementations for heterogeneous master-worker platforms. These implementations are based on the data allocation and dynamic load balancing strategies. The first implementation is the dynamic master - worker with allocation of vectors where the master distributes vectors (data) and computations to the workers whereas the second one is the dynamic master - worker with allocation of vector pointers where the vectors are supposed to be replicated among participating resources beforehand and the master distributes computations to the workers. We also report a set of numerical experiments on a heterogeneous platform where computational resources have different computing powers. Also, the workers are connected to the master by links of same capacities. The obtained experimental results demonstrate that the dynamic allocation of vector pointers achieve better performance than the original one for computing dot product computation.
\end{abstract}

The paper also presents and verifies an accurate timing model to predict the performance of the proposed implementations on clusters of heterogeneous workstations. Through this model the viability of the proposed implementa-

Received: December 18, 2012

(C) 2013 Academic Publications, Ltd.

$\S$ Correspondence author url: www.acadpubl.eu 
tions can be revealed without the extra effort that would be needed to carry out real testing.

AMS Subject Classification: 65F30, 65Y05, 68M14, 68M20, 68W10

Key Words: dot product, cluster of heterogeneous workstations, Message passing interface, parallel algorithms, performance prediction

\section{Introduction}

Dot product is a key computational kernel in many scientific applications of research such as linear algebra, signal processing, physics, digital control and graph theory. In mathematics, the dot product, also known as the scalar product, is an operation which takes two vectors over the real numbers $R$ and returns a real-valued scalar quantity [1]. It is the standard inner product of the Euclidean space. In Euclidean geometry, the dot product, length, and angle are related: For a vector $a, a * a$ is the square of its length, and more generally, if $b$ is another vector

$$
a * b=|a| *|b| \cos \theta
$$

where

- $|a|$ and $|b|$ denote the length (magnitude) of $a$ and $b$,

- $\theta$ is the angle between them.

Since $|a| \cos (\theta)$ is the scalar projection of $a$ onto $b$, the dot product can be understood geometrically as the product of the length of this projection and the length of $b$.

In linear algebra, the dot product of two vectors $a=\left[a_{1}, a_{2}, \ldots, a_{n}\right]$ and $b=\left[b_{1}, b_{2}, \ldots, b_{n}\right]$ is defined as follows [1]:

$$
a * b=\sum_{i=1}^{n} a_{i} * b_{i}=a_{1} * b_{1}+a_{2} * b_{2}+\ldots+a_{n} * b_{n}
$$

where $\Sigma$ denotes summation notation and $n$ is the size of the vector.

Dot products can be used as a basic core in many applications ranging from scientific computations to commercial applications in data mining. Typical scientific computations utilizing iterative solvers use repeated matrix-vector operations. These can be viewed as dot products of vectors. In distributed data mining applications, dot products take the form of counting operations [24]. The demand for such applications is expected to increase significantly and 
with it, the need for efficiency. As a result, with widespread use of distributed platforms, these operations are time consuming across very large amounts of data or a large number of vectors.

To address the computational requirements introduced by linear algebra applications, several efforts have been directed towards the incorporation of high performance computing models in large scale scientific computations. With the aim of creating a cost-effective parallel computing system from commodity components to satisfy specific computational requirements for the linear algebra operations, developed the concept of Beowulf cluster [11]. The processing power offered by such commodity systems has been traditionally employed in scientific computing applications from very large data archives. Although most parallel techniques and systems for scientific computing employed by several researchers during the last decade have been chiefly homogeneous in nature, a current trend in the design of systems for the large scale scientific computations, relies on the utilization of highly heterogeneous computing platforms. Unlike traditional homogeneous systems, heterogeneous clusters are composed of workstations running at different speeds. As a result, traditional parallel linear algebra algorithms, which distribute computations evenly across the different workstations, cannot balance the load of different-speed workstations in heterogeneous clusters as faster workstations will quickly perform their portions of computation and will have to wait for slower ones at points of synchronization. Therefore, a natural solution to the problem of heterogeneous computing is to distribute data across workstations unevenly or to distribute data to idle workstations dynamically, so that each workstation performs the volume of computation proportional to its speed. Heterogeneous computing research $[28,26,29]$ has shown that, with careful job scheduling, off-the-shelf heterogeneous clusters can realize a very high level of aggregate performance. Subsequently, it is expected that these clusters will represent an useful tool for the scientific community devoted to the scientific computations. Due to the recent incorporation of heterogeneous computing to linear algebra [18], significant opportunities to exploit such techniques are available in the dot product computation.

In this paper, we propose parallel implementations for solving the dot product computation on heterogeneous clusters. Our goal is to study data allocation and workload balancing issues in order to improve the parallel execution of the dot product computation onto a cluster of heterogeneous workstations. It is not our intention to prove that the use of a cluster is better than the use of a specific parallel machine nor to make any modification to the dot product computation. Indeed, we adopt two realistic application scenarios, where one 
scenario the input vector files are read from a fixed repository (such as a disk on a data server) and the other one the input vector files are read from the local repositories (such as the local disks on the worker clients). In both scenarios the computations are delegated to available computational resources, and results will be returned to the master. In both cases we call for a master - worker paradigm where the master assigns computations to other resources, the workers. Typically, our approaches are useful in the context of speeding up MATLAB, Octave, SCILAB or R clients running on a server (which acts as the master and initial repository of files) or speeding up clients running on a cluster of servers (which act as workers and replication of vector files). This presented work exploit the parallelism similar to the Map-Reduce framework [15] or the Hadoop project [44] where the goal is to perform grep and more interesting data mining on very large amounts of data.

To summarize, the target heterogeneous master-worker platform is composed of computational resources or workers with different computing powers, and workers are connected to the master by links of same capacities. The main contributions of this paper are twofold:

- On the practical side, we have proposed and evaluated two parallel implementations of dot product computation on heterogeneous master - worker platforms. These implementations are based on the data allocation and dynamic load balancing strategies.

- On the theoretical side, we have proposed a performance model to predict the performance of the proposed implementations on arbitrary cluster of heterogeneous workstations. Further, it has been checked against experimental results and it is shown that the model is able to predict the parallel performance accurately.

The rest of the paper is organized as follows. In the next section, we review some related work for linear algebra computations and discuss some parallel alternatives. In Section 3 we briefly present heterogeneous computing platform and the metrics. Two parallel dot-product implementations for heterogeneous platforms are presented in Section 4, followed by a performance estimation model in Section 5. Next, some MPI experimental results are presented in Section 6. Finally, the paper is concluded by a recount of obtained results. 


\section{Related Work}

There are many works available in the literature concerned with linear algebra or more generally, matrix product algorithms and parallelization issues. However parallelization of dot product has not yet been studied carefully, especially in the data allocation and load balancing context.

To the best of our knowledge, there are only a few published works with a data allocation context and the emphasis on data allocation we are concerned here. Most works have focused on specific parallel architectures and parallel and distributed data allocation issues are sometimes even ignored.

The efficient implementation of dot product and matrix computations on parallel computers is an issue of prime importance when providing such systems with scientific software libraries $[4,13,14,16,17]$ or applications which use the dot product computation as data processing mechanism [37, 38, 3, 10, 22, 23, 32]. Consequently, considerable effort has been devoted in the past to the development of efficient parallel matrix algorithms and this will remain a task in the future as well. This is mainly due to evolutionary architectures of parallel and distributed computers such as 1D- and 2D-systolic arrays, 2D-meshes, 2-D tori, and hypercubes, as well as the shared memory model and distributed memory model. Many parallel algorithms have been designed, implemented, and tested on different parallel computers or clusters of homogeneous workstations for matrix computations $[19,40,21,43]$. It is possible that most of the reported parallel algorithms used in practice are based on parallel multicomputers where the processors are interconnected in a two dimensional mesh. These algorithms may be roughly classified as "broadcast and shift algorithms" initially presented in [19] and "align and shift algorithms", initially presented in [12]. Both kinds of algorithms are described in terms of two-dimensional processor grids where each processor holds a large consecutive block of data.

Several distributed implementations BLACS, ScaLAPACK, PUMMA, SUMMA and LAPACK $[2,4,13,14,16,17,20,30]$ have been successfully adapted the broadcast based algorithms to the point-to-point interconnection of two dimensional torus. All of them aim to obtain the best performance for the whole algorithm by implementing broadcast over the point-to-point communication links of distributed memory parallel computers.

However, current parallel architectures typically take the form of heterogeneous clusters, which are composed of heterogeneous computing resources, interconnected by a sparse network; there are no direct links between any pair of workers but there are links only from master to workers [18]. Recently, several parallel algorithms for similar problems of linear algebra have been pro- 
posed and implemented on cluster of heterogeneous workstations $[5,6,7,27,9$, $25,34,40,18]$. They use different distribution schemes and redesigned several parallel matrix algorithms designed for traditional parallel computers (i.e., 2D processor grid strategies) to heterogeneous clusters.

Further, the general efficient algorithms for the master-worker paradigm on heterogeneous clusters for scientific computations have been widely developed in $[8,33,18]$. They use different data allocation and load balancing strategies for efficient parallel execution of the matrix product computations on heterogeneous clusters. Distributing the computations (together with the associated data) can be performed either dynamically or statically, or a mixture of both. Some simple schedulers are available, but they use naive mapping strategies such as master-worker techniques or paradigms based upon the idea "use the past to predict the future".

A similar thread of work, although in a different context, deals with reconfigurable architectures, either pipelined bus systems [31] or FPGAs [41]. In the latter approach, tradeoffs must be found to optimize the size of the on-chip memory and the available memory bandwidth, leading to partitioned algorithms that re-use data intensively.

\section{Heterogeneous Computing Platform}

A heterogeneous network (HN) can be abstracted as a connected graph $\mathrm{HN}(\mathrm{M}, \mathrm{C})$, where

- $\mathrm{M}=\left\{M_{1}, M_{2}, \ldots, M_{p}\right\}$ is set of heterogeneous workstations ( $p$ is the number of workstations). The computation capacity of each workstation is determined by the power of its CPU, I/O and memory access speed.

- $\mathrm{C}$ is standard interconnection network for workstations, such as Fast Ethernet or an ATM network, where the communication links between any pair of the workstations have the same bandwidth.

Based on the above definition, if a cluster consists of a set of identical workstations, the cluster is homogeneous.

\subsection{Metrics}

Metrics help to compare and characterize parallel computer systems. Metrics cited in this section are defined and published in previous paper [42]. They can be roughly divided into characterization metrics and performance metrics. 


\subsubsection{Characterization Metrics}

To compute the power weight among workstations an intuitive metric is defined as follows:

$$
W_{i}(A)=\frac{\min _{j=1}^{p}\left\{T\left(A, M_{j}\right)\right\}}{T\left(A, M_{i}\right)}
$$

where $\mathrm{A}$ is an application and $\mathrm{T}\left(\mathrm{A}, M_{i}\right)$ is the execution time for computing $\mathrm{A}$ on workstation $M_{i}$. Formula 3 indicates that the power weight of a workstation refers to its computing speed relative to the fastest workstation in the network. The value of the power weight is less than or equal to 1 . However, if the cluster of workstations is homogeneous then the values of the power weights are equal to 1 .

To calculate the execution time of a computational segment, the speed, denoted by $S_{f}$ of the fastest workstation executing basic operations of an application is measured by the following equation:

$$
S_{f}=\frac{\Theta(c)}{t_{c}}
$$

where $c$ is a computational segment, $\Theta(c)$ is a complexity function which gives the number of basic operations in a computational segment and $t_{c}$ is the execution time of $c$ on the fastest workstation in the network.

Using the speed of the fastest workstation, $S_{f}$, we can calculate the speeds of the other workstations in the system, denoted by $S_{i}(i=1, \ldots, p)$, using the computing power weight as follows:

$$
S_{i}=S_{f} * W_{i}, i=1, \ldots, p, \text { and } i \neq f
$$

where $W_{i}$ is the computing power weight of $M_{i}$. So, by equation 5 , the execution time of a segment $c$ across the heterogeneous network $\mathrm{HN}$, denoted by $T_{c p u}(\mathrm{c}, \mathrm{HN})$, can be represented as

$$
T_{c p u}(c, H N)=\frac{\Theta(c)}{\sum_{i=1}^{p} S_{i}}
$$

where $\sum_{i=1}^{p} S_{i}$ is the computational capacity used which is obtained by summing the individual speeds of the workstations. Here, $T_{c p u}$ is considered the required CPU time for the segment. Furthermore, substituting $S_{i}=1$ in above equation for dedicated cluster of homogeneous workstations, the execution time of a segment $c$ returns to the conventional form:

$$
T_{\text {cpu }}(c, H N)=\frac{\Theta(c)}{p}
$$




\subsubsection{Performance Metrics}

Speedup is used to quantify the performance gain from a parallel computation of an application A over its computation on a single machine on a heterogeneous network system. The speedup of a heterogeneous computation is given by:

$$
S P(A)=\frac{\min _{j=1}^{p}\left\{T\left(A, M_{j}\right)\right\}}{T(A, H N)}
$$

where $\mathrm{T}(\mathrm{A}, \mathrm{HN})$ is the total parallel execution time for application $\mathrm{A}$ on $\mathrm{HN}$, and $\mathrm{T}\left(\mathrm{A}, M_{j}\right)$ is the execution time for $\mathrm{A}$ on workstation $M_{j}, j=1, \ldots, p$.

Efficiency or utilization is a measure of the time percentage for which a machine is usefully employed in parallel computing. Therefore, the utilization of parallel computing of application A on a dedicated heterogeneous network is defined as follows:

$$
E=\frac{S P(A)}{\sum_{j=1}^{p} W_{j}}
$$

The previous formula indicates that if the speedup is larger than $\sum_{j=1}^{p} W_{j}$, the system computing power, the computation presents a superlinear speedup in a dedicated heterogeneous network. Further, substituting $W_{j}=1$ in above equation for dedicated cluster of homogeneous workstations, the utilization returns to the traditional form:

$$
E=\frac{S P(A)}{p}
$$

\section{Parallel Dot - Product Implementations}

Given any two vectors $a$ and $b$ of size $n$, their dot product $a * b$ can be computed in $O(n)$ time. In this section describes two parallel dot product algorithms that will be compared in this study. These algorithms are based on the master worker programming paradigm and they take into account two criteria: the dynamic load balancing strategy and the data allocation strategy. In dynamic load balancing strategy, the vectors are partitioned into small subvectors and these subvectors are assigned dynamically to idle workstations in order to keep all the workstations busy. The size of each subvector is $s b$ successive elements. This block size is an important parameter which can affect the overall performance. More specifically, this parameter is directly related to the $\mathrm{I} / \mathrm{O}$ and communication factors. For this reason, we use a block-oriented approach to partition the two vectors $a$ and $b$ into blocks of size $s b$. 
On the other hand, the data allocation strategy also is divided into two categories: allocation of subvectors and allocation of subvector pointers. In allocation of subvectors, the subvectors that are obtained by dynamic load balancing strategy are distributed to corresponding workstations. In allocation of subvector pointer, some master workstation of the cluster has a text pointer that shows the current position in the vectors and the master distributes the subvector pointers instead of the subvectors to corresponding workers in order to reduce the communication overhead. Dynamic allocation of subvectors and dynamic allocation of subvector pointers are presented in next subsections.

\subsection{Dynamic Allocation of Subvectors}

Our dynamic allocation of subvectors is based on the following assumptions: First, the number of workstations in the cluster is denoted by $p$ and we assume that $p$ is power of 2. Further, the workstations are numbered from 1 to $p$. Second, we load into the local disk of the master workstation all subvectors (or blocks) of the vectors $a$ and $b$. The algorithm of the dynamic allocation of subvectors that is called PD1 is made of two parts: Algorithm 1 outlines the program of the master, while Algorithm 2 is the program of each worker. We must note that the of $f$ set is a pointer that shows the current position in the file. Further, the master terminates when there are not any blocks of the vectors $a$ and $b$. Note that in order to terminate, the number of tasks outstanding in the workers is counted (active). It is also possible simply to count the number of results returned.

\subsection{Dynamic Allocation of Vector Pointers}

Our dynamic allocation of subvector pointers is based on the following assumptions: First, we load into the local disks of all worker workstations all blocks of the vectors $a$ and $b$ and second, the master workstation has a pointer that shows the current position in the vectors $a$ and $b$ and it distributes the subvector pointers in order to reduce the communication overhead of the PD1 implementation. The algorithm of the dynamic allocation of subvector pointers that is called PD2 is made of two parts: Algorithm 3 outlines the program of the master, while Algorithm 4 is the program of each worker. 

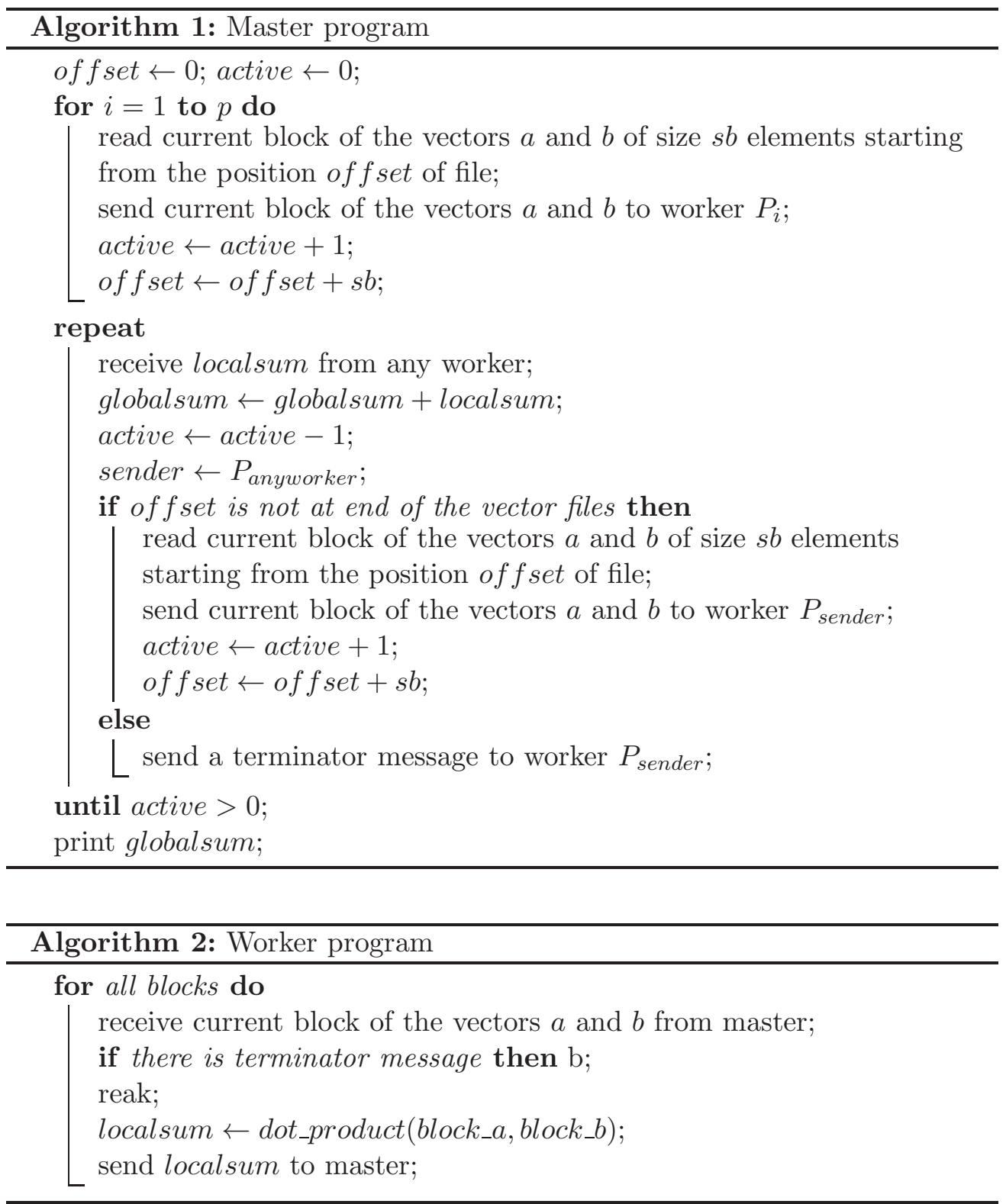

\section{Performance Results}

Performance prediction models can be greatly contribute to the parallel computing. In this section we present two time prediction models for the PD1 and 


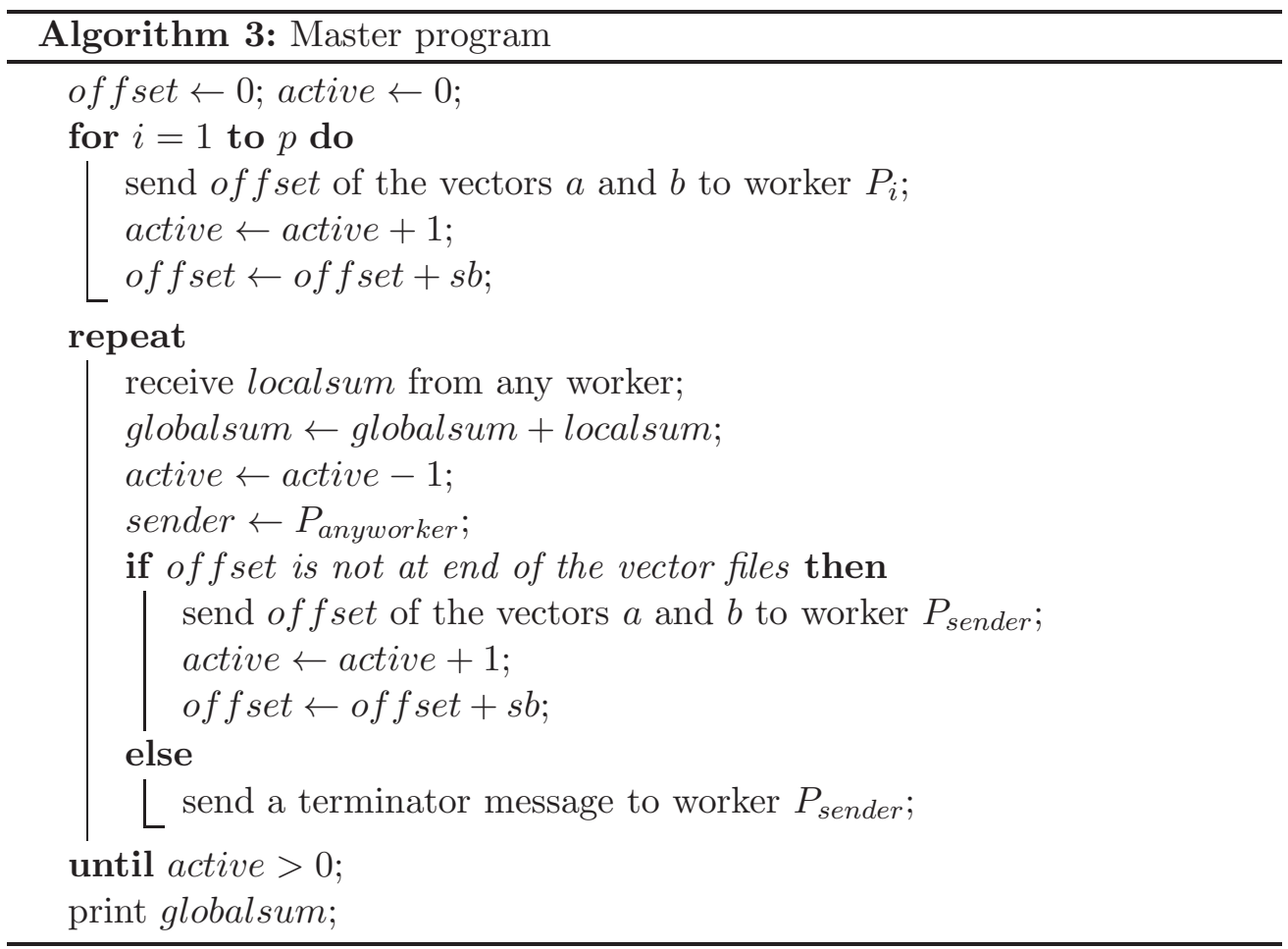

Algorithm 4: Worker program

for all blocks do

receive of $f$ set from master;

if there is terminator message then $\mathrm{b}$;

reak;

read current block of the vector $a$ and $b$ from the local disk $s b$

elements of the file starting from position of $f$ set;

localsum $\leftarrow$ dot_product $($ block_a,block_b);

send localsum to master;

PD2 implementations discussed above. One of the key qualities of this model stems from the fact that machine and vectors distribution characteristics are separated from the timing parameters. The model can be used to predict the performance of two parallel implementations on arbitrary machine clusters and using arbitrary data distributions. Therefore, the performance models for two implementations are general enough to cover performance evaluation of both 
homogeneous and heterogeneous computations in a dedicated cluster of workstations. So, we consider the homogeneous computing as a special case of heterogeneous computing. Two performance prediction models are presented in next subsections.

\subsection{Performance Model for the Dynamic Allocation of Subvectors}

We give the execution time for each step of the PD1 implementation. Therefore, the execution time can be broken up into four terms:

- $T_{a}$ : It is the total I/O time to read the elements of the vectors $a$ and $b$ into several blocks of size $s b *$ sizeof (int) bytes from the local disk of the master workstation. The $s b$ is the number of elements of the block. Therefore, the master reads $2 * n *$ sizeof $($ int $)$ bytes totally of the vectors $a$ and $b$. Then, the time $T_{a}$ is given by:

$$
T_{a}=\frac{2 * n * \operatorname{sizeof}(\text { int })}{\left(S_{I / O}\right)_{\text {master }}}
$$

where $\left(S_{I / O}\right)_{\text {master }}$ is the I/O speed of the master workstation.

- $T_{b}$ : It is the total communication time to send all blocks of the vectors $a$ and $b$ to all workers. The size of each block is $s b * \operatorname{sizeof}($ int $)$ bytes. Therefore, the master sends $2 * n *$ sizeof (int) bytes totally. Then, the time $T_{b}$ is given by:

$$
T_{b}=\frac{2 * n * \operatorname{sizeof}(\text { int })}{S_{\text {comm }}}
$$

where $S_{\text {comm }}$ is the communication speed.

- $T_{c}$ : It is the average computation time across the cluster. Each worker performs a dot product between the block of the vector $a$ and the block of the vector $b$ with size $s b * s i z e o f(i n t)$ bytes. It requires $s b$ steps. Then, the time $T_{c}$ is given by:

$$
T_{c}=\frac{\left(\left\lceil\frac{n}{s b}\right\rceil-p\right)(\text { sb* sizeof }(\text { int }))}{\sum_{j=1}^{p}\left(S_{\text {comp }}\right)_{j}}+\max _{j=1}^{p}\left\{\frac{\text { sb*sizeof }(\text { int })}{\left(S_{\text {comp }}\right)_{j}}\right\}
$$

where $\Sigma_{j=1}^{p}\left(S_{\text {comp }}\right)_{j}$ is the computation capacity of the cluster (homogeneous or heterogeneous) when $p$ workstations are used. We include the second max term in the equation 13 which defines the worse load imbalance at the end of the execution when there are not enough blocks of the vectors left to keep all the workstations busy. 
- $T_{d}$ : It includes the communication time to receive $\left\lceil\frac{n}{s b}\right\rceil$ results from all workers. Each worker sends the local sum back $1 * \operatorname{sizeof}($ int $)$ bytes. Therefore, the master will receive $\left\lceil\frac{n}{s b}\right\rceil *$ sizeof (int) bytes totally. Therefore, the time $T_{d}$ is given by:

$$
T_{d}=\frac{\left\lceil\frac{n}{s b}\right\rceil * \operatorname{sizeof}(\text { int })}{S_{\text {comm }}}
$$

where $S_{\text {comm }}$ is the communication speed.

Further, we note that this dynamic implementation in practice there is parallel communication and computation and this reason we take the maximum value between the communication time i.e., $T_{b}+T_{d}$ and the computation time i.e., $T_{c}$. Therefore, the total execution time of our dynamic implementation, $T_{p}$, using $p$ workstations, is given by:

$$
T_{p}=T_{a}+\max \left\{T_{b}+T_{d}, T_{c}\right\}
$$

\subsection{Performance Model for the Dynamic Allocation of Vector Pointers}

We give the execution time for each step of the PD2 implementation. Therefore, the execution time can be broken up into four terms:

- $T_{a}$ : It is same with the time $T_{b}$ of the previous implementation but this term includes the total communication time to send $\left\lceil\frac{n}{s b}\right\rceil$ vector pointers instead of blocks of elements of the vectors $a$ and $b$ to all workers. Therefore, the time $T_{a}$ is given by:

$$
T_{a}=\frac{\left\lceil\frac{n}{s b}\right\rceil * \operatorname{sizeof}(\text { int })}{S_{\text {comm }}}
$$

where $S_{\text {comm }}$ is the communication speed.

- $T_{b}$ : It is the total I/O time to read the vectors $a$ and $b$ into several blocks of size $s b * \operatorname{sizeof}($ int $)$ bytes from the local disks of the worker workstations. We note that each worker reads from the local disk $s b * s i z e o f($ int $)$ bytes of the vector $a$ and other $s b * \operatorname{sizeof}($ int $)$ bytes of the vector $b$ starting from the pointer that receives. Then, the time $T_{b}$ is given by:

$$
T_{b}=\frac{\left(2 *\left\lceil\frac{n}{s b}\right\rceil-p\right)(s b * \operatorname{sizeof}(\text { int }))}{\Sigma_{j=1}^{p}\left(S_{I / O}\right)_{j}}+\max _{j=1}^{p}\left\{\frac{s b * \operatorname{sizeof}(\text { int })}{\left(S_{I / O}\right)_{j}}\right\}
$$


where $\Sigma_{j=1}^{p}\left(S_{I / O}\right)_{j}$ is the I/O capacity of the cluster (homogeneous or heterogeneous) when $p$ workstations are used. We include the second $\max$ term in the equation 17 which defines the worse load imbalance at the end of the execution when there are not enough blocks of the vectors left to keep all the workstations busy.

- $T_{c}$ : It includes the average computation time across the cluster. The amount of this time is similar to the time $T_{c}$ of the previous implementation.

- $T_{d}$ : It includes the communication time to receive the results of the local dot product from all workers. The amount of this time is same with the time $T_{d}$ of the previous implementation.

We take the maximum value between the communication time i.e., $T_{a}+T_{d}$ and the computation time i.e., $T_{b}+T_{c}$, since in this implementation there is parallel communication and computation. Therefore, the total execution time of our dynamic implementation, $T_{p}$, using $p$ workstations, is given by:

$$
T_{p}=\max \left\{T_{a}+T_{d}, T_{b}+T_{c}\right\}
$$

\section{Experimental and Theoretical Results}

In this section, the performance of the PD1 and PD2 implementations are investigated on a cluster of heterogeneous workstations and we conduct a variety of experiments to validate our performance models with experimental results.

\subsection{Experimental Environment}

The target platform for our experimental study is a cluster of 16 heterogeneous workstations running Linux operating system (RedHat 7.1). More specifically, the cluster consists of 8 Pentium II $266 \mathrm{MHz}$ with $64 \mathrm{MB}$ RAM and 8 Pentium $166 \mathrm{MHz}$ with $32 \mathrm{MB}$ RAM. The communication between workstations is performed through a 100Mbps Fast Ethernet network. The configuration of the heterogeneous cluster was as follows: 8 Pentium II $266 \mathrm{MHz}$ and 8 Pentium 166 $\mathrm{MHz}$. The middleware of the clusters is ROCKS of NPACI [36]. The MPI implementation used on the network is MPICH version 1.2. The employed cluster environment is a typical installation with dedicated local area network. In order to avoid pointless scenarios, the experiments were conducted after midnights 
where no unknown loads on the workstations. Furthermore, unnecessary time stealing daemons were blocked at the time of experimentation.

MPI is a software for message passing, proposed as a standard by a broad committee of vendors, implementers, and users [39]. MPI is portable and flexible software that is used widely. It is considered as a standard for writing message-passing programs in which higher level routines and abstractions are built upon lower level message passing routines.

In our experiments we implemented PD1 and PD2 in C programming language using the MPI library [35, 39]. These two implementations were tested using vectors of orders ranging from 512 up to 2048 with block size of 2, 4 and 8 elements. We must note that we selected these vector sizes so that they can be stored in workstations memories. The experiments have been repeated using 2, 4, 8 and 16 workstations for both implementations.

\subsection{Experimental Results}

Figure 1 shows the real execution times and speedup curves for MPI-based PD1 on block size of 2, 4 and 8 elements and 2, 4, 8 and 16 workstations with vector orders ranging from 512 to 1024 elements. As can be seen from Figure that the execution time is increased as the number of workstations and the vector size are increased due to the high communication cost. Furthermore, as the number of workstations increases, the speedup curves of the PD1 implementation appear to deviate from the ideal ones. More specifically, from extensive experimental study has been shown that there are two basic reasons for the performance degradation of the PD1 implementation. The primary factor affecting the performance of the implementation is high communication requirements between master and workers for distributing the vectors. Another reason for the performance degradation is due to the high cost of reading of data from the local disk of the master workstation for small values of block size. The I/O time could be improved when the vector is read and partitioned in chunks of large size. However, the execution time of the PD1 implementation is improved slightly for large values of block size because low communication requirements are obtained whereas there is worst performance for very small values of block size (i.e., 2, 4) because produce a poorly balanced load. Therefore, we conclude that the block size is an important parameter which can affect the I/O and communication costs of the total execution time. So, the low communication and $\mathrm{I} / \mathrm{O}$ cost is obtained for large values of block size and high communication and $\mathrm{I} / \mathrm{O}$ cost is obtained for small values of block size.

Figure 2 shows the real execution times and speedup curves for MPI-based 

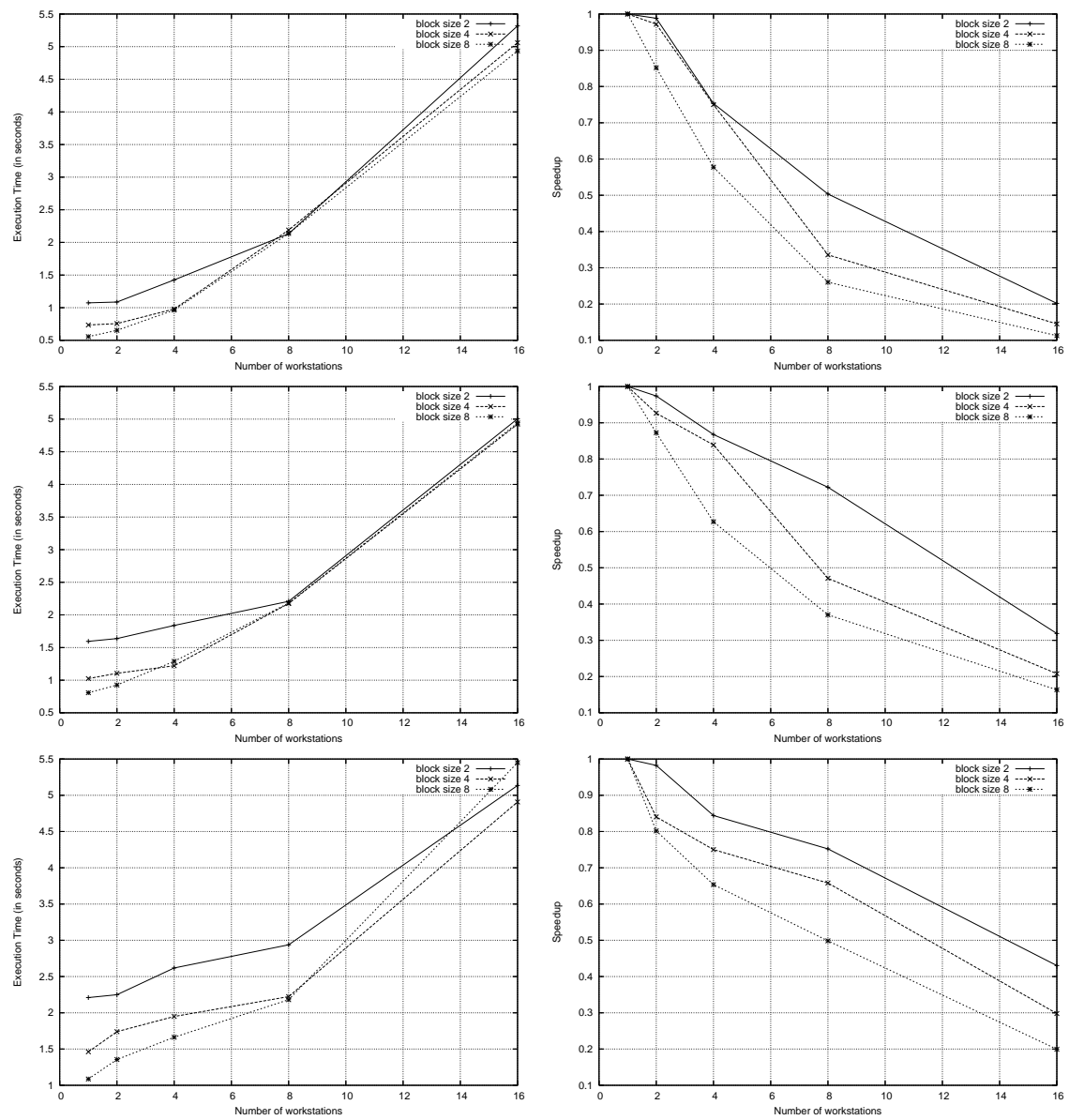

Figure 1: The real execution times (left) and speedup curves (right) for MPI based PD1 implementation for vector sizes of 512, 1024 and 2048

PD2 on block size of 2, 4 and 8 elements and 2, 4, 8 and 16 workstations with vector orders ranging from 512 to 1024 elements. We observe that the execution time is decreased as the number of workstations and the block size are increased. Further, the speedup curves of the PD2 implementation are increased linearly as the number of workstations is increased. We note that the speedup curves of the implementation are very close to the ideal system computing power, $\Sigma_{j=1}^{p} W_{j}$ for all number of workstations. Although the implementation runs faster on a larger number of workstations, the gain in the speedup factor is slower. For instance, the difference in execution time between 8 and 16 workstations is smaller than the difference between 4 and 8 workstations. This is due to the dominance of 
increased communication cost over the reduced in computation cost. Moreover, we must note that the block size is an important parameter which can be affect the I/O time only if the total execution time is compared to the PD1 implementation. In other words, high cost of reading of data from the local disk of the workstations is obtained for small values of block size whereas low cost of reading of data is obtained for large values of block size. In this parallel implementation, the block size does not affect the communication time since the communication requirements include the distribution vector pointers instead of chunks of vectors. Another parameter which can affect the performance of parallel implementation is the vector size. From Figures, we can see that the execution time and the speedup curves of parallel implementation are increased slightly as the vector size is increased. This occurs, because when the vector size is large enough, due to lot of arithmetic computations performed in parallel at each workstation. However, the communication overhead between the master workstation and worker workstations is not very significant. Therefore, the ratio of the computation time to communication time is high enough

The ranking of the parallel implementations is quite different when we look at the Figures 1 and 2. First, we have the PD2 implementation which gives good performance with low communication and I/O requirements. Finally, we have the PD1 implementation which do not achieve an efficient performance and thus achieves very high communication requirements.

\subsection{Theoretical Results}

The final experiment was conducted to verify the correctness of the proposed timing models for PD1 and PD2 implementations. The performance models of equations 15 and 18 are plotted in Figures 3 and 4 using MPI time parameters, respectively. In order to get these estimated results, we have determined the values of the power weights and the values of the speeds $S_{I / O}$ and $S_{\text {comp }}$ of the fastest workstation. The computing power weight provides an average performance reference by including the heterogeneity of network systems. The power weight is a combination measurement of a program and a cluster system. We consider the effects of memory size on the execution of the two implementations of dot product computation, which is a major architecture performance factor.

In our experiments, the execution timing results of dot product computations on the network system were measured using different vector sizes. The performance is directly related to the memory size in each workstation. The average computing power weights of the different types of workstations based on formula 3 were measured when the matrix size does not exceed the mem- 

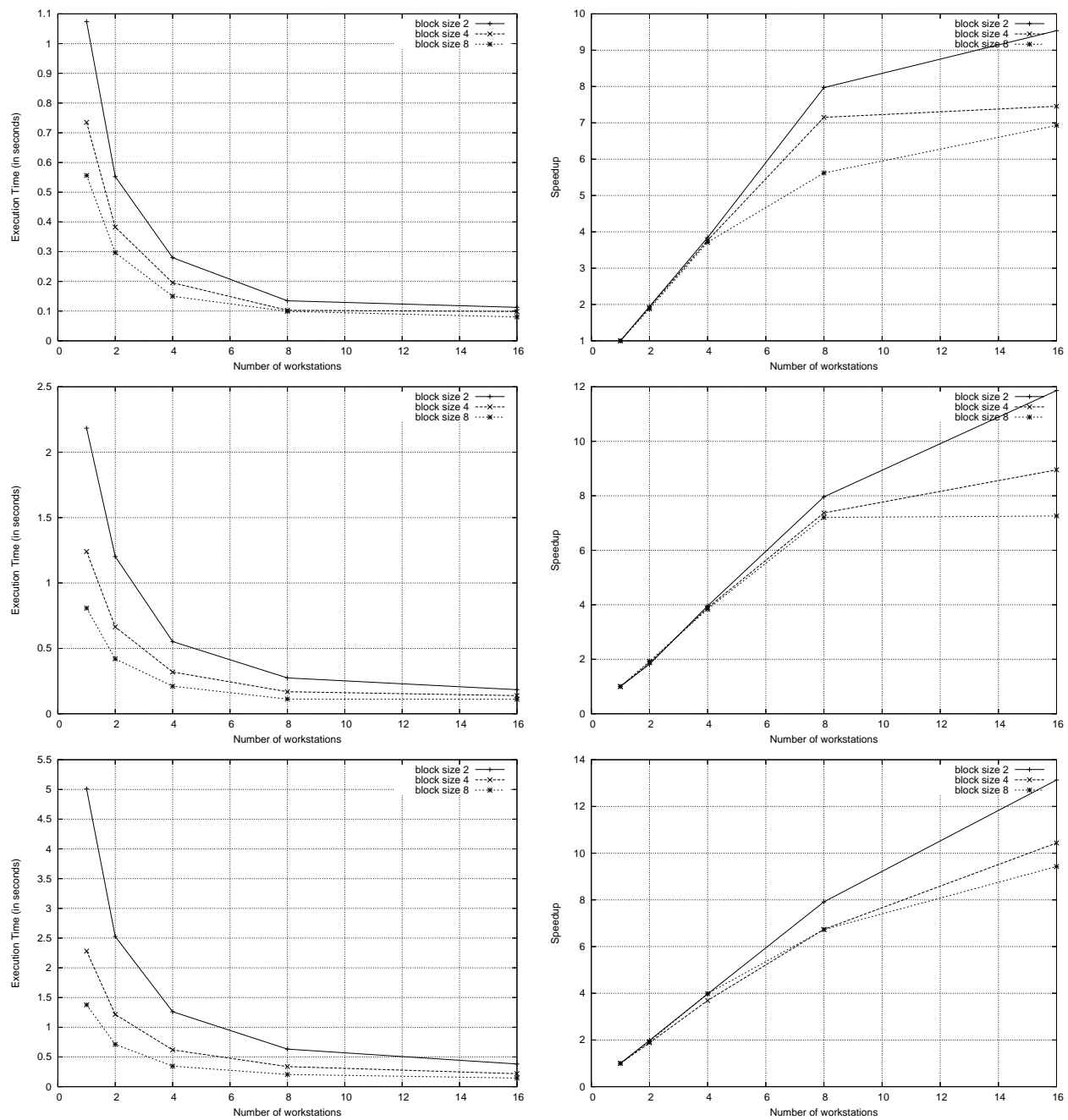

Figure 2: The real execution times (left) and speedup curves (right) for MPI based PD2 implementation for vector sizes of 512, 1024 and 2048

ory bound of any machine in the system in order to keep the power weights constant.

Further, the average speeds $S_{I / O}$ and $S_{\text {comp }}$ of the fastest workstation for all block sizes $(b=2,4$ and 8$)$, executing the dot product computation are critical parameters for predicting the CPU demand times of computational segments on other workstations. The speeds were measured for different vector sizes and averaged by formula 4 . To obtain an accurate estimate, the communication 
speed was measured for different vector sizes and block sizes and it has been averaged over one hundred tests. Therefore, the average speeds $S_{I / O}$ and $S_{c o m p}$ are as follows, $S_{I / O}=4758500 \mathrm{ints} / \mathrm{sec}, S_{c o m p}=5710072 \mathrm{ints} / \mathrm{sec}$ for two implementations. Finally, the communication speed was measured for different vector sizes and block sizes as follows, $S_{\text {comm }}=41214492 \mathrm{ints} / \mathrm{sec}$. We must note that we calculated the speeds $S_{I / O}$ and $S_{\text {comp }}$ of the fastest workstation in order to calculate the speeds of the other workstations in the cluster by formula 5 . Using the speeds $\left(S_{I / O}\right.$ and $\left.S_{\text {comp }}\right)$ of all workstation in the cluster, we can get the estimated results by the performance models of equations 15 and 18 .

As can be seen from Figures 3 and 4, the estimated execution times are quite close to the real ones. This verifies that the proposed timing models for PD1 and PD2 fairly accurate, and hence it provides a means to test the viability of PD1 and PD2 on any cluster without taking the burden of real testing. However, there are small differences between the experimental and theoretical estimated results in the PD1 and PD2 implementations. This occurs because our performance model did not take into account the idle time of the master. In other words, when master wants to give some load to a worker but the worker is busy doing some computation.

\section{Conclusions}

In this paper we proposed and evaluated two parallel implementations of the dot product computation using the master - worker programming paradigm. The implementations have been tested on a cluster of 16 heterogeneous workstations connected together using $100 \mathrm{Mbps}$ Fast Ethernet network. The practical results obtained have helped us to evaluate the parameters (such as the number of workstations, block size and vector size) that must be taken into account. If possible, these should be tailored in order to obtain the best performance for running time and throughput in this scientific computing context. Moreover, the obtained experimental results show an efficient performance in favor of PD2 implementation over the PD1 implementation. The key benefits that have been the driving force in implementing the PD2 are: linear time complexity (with sufficiently large number of workstations) and low communication overhead.

Further, we have introduced an effective performance prediction model and framework, which can help to users to predict the execution time and similar performance metrics of the proposed dot product implementations for two kinds of cluster (homogeneous and heterogeneous). It has been shown that the 

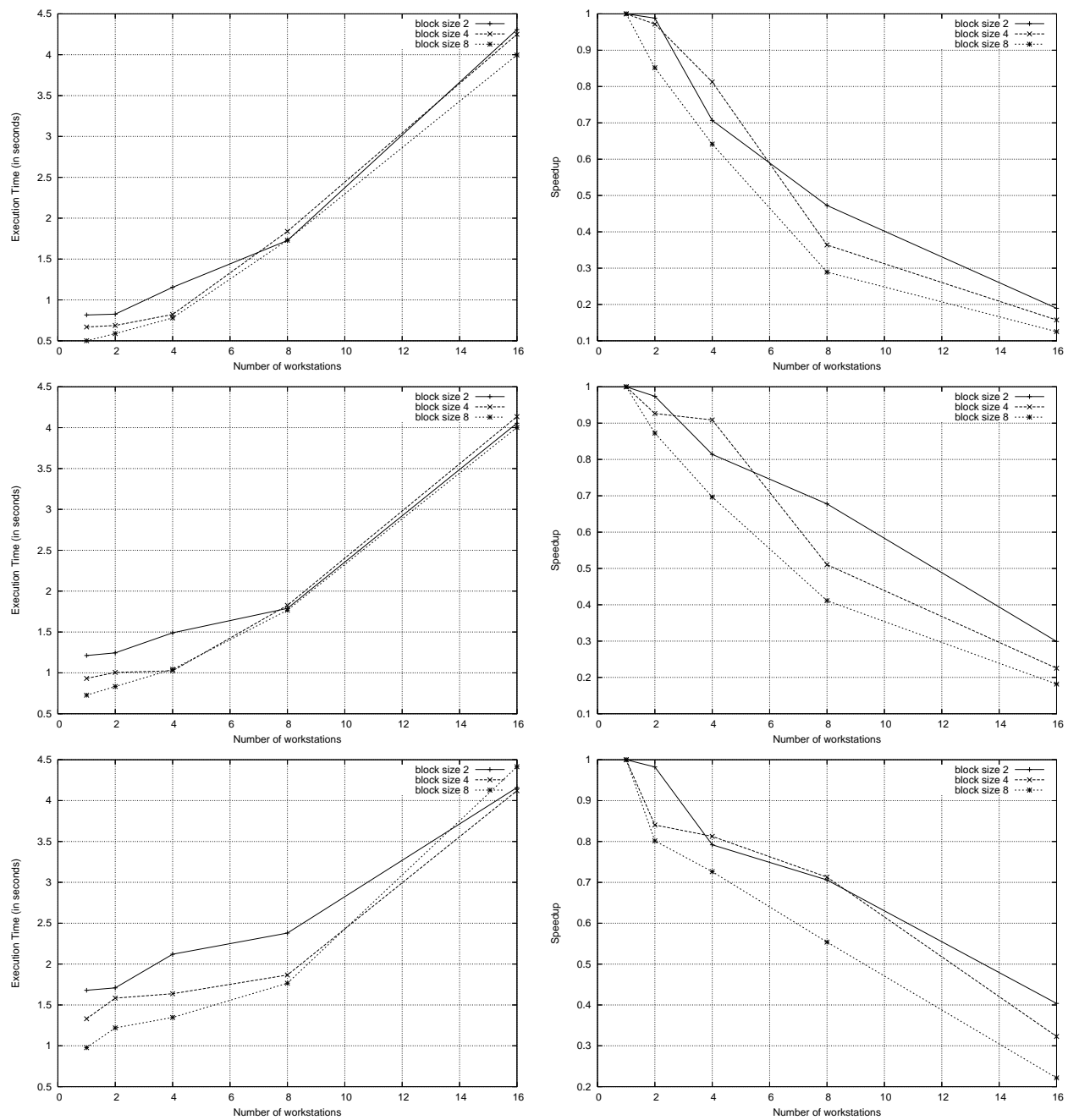

Figure 3: The estimated execution times (left) and speedup curves (right) for MPI based PD1 implementation for vector sizes of 512, 1024 and 2048

performance model is able to predict the parallel execution time of parallel dot product implementations accurately.

It would be interesting to assess the performance of our parallel implementations with respect to memory and communication heterogeneity. Further, it would be interesting to implement the dot product computation for large vector sizes that can not be stored in worker memories but only on hard disk. 

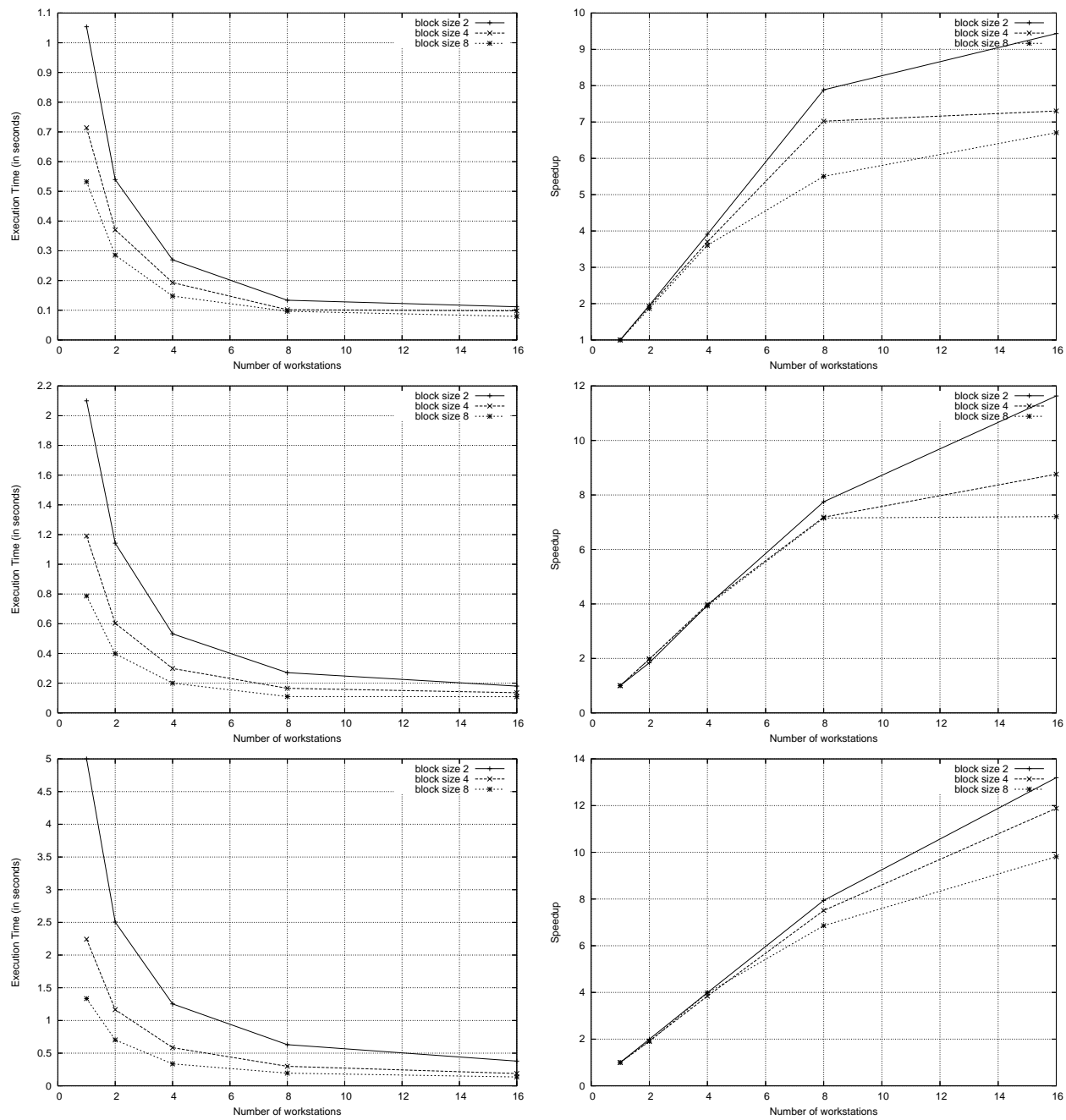

Figure 4: The estimated execution times (left) and speedup curves (right) for MPI based PD2 implementation for vector sizes of 512, 1024 and 2048

\section{References}

[1] Dot Product, http://en.wikipedia.org/wiki/Dot_product, 2012.

[2] R.C. Agarwal, F.G. Gustavson, M. Zubair, A high-performance matrix multiplication algorithm on a distributed-memory parallel computer using 
overlapped communication, IBM Journal of Research and Development, 38 (1994), 673-681.

[3] P. Alonso, G. Flores-Becerra, A.M. Vidal, Sequential and Parallel Algorithms for the Inverse Toeplitz Singular Value Problem, In: Proceedings of the International Conference on Scientific Computing 2006 (CSC 2006) (2006), 91-96.

[4] E. Anderson, Z. Bai, C. Bischof, J. Demmel, J. Dongarra, J. Du Croz, A. Greenbaum, S. Hammarling, A. McKenney, S. Ostrouchov, D. Sorensen, LAPACK Users' Guide, SIAM, Philadelphia (1992).

[5] O. Beaumont, V. Boudet, A. Petitet, F. Rastello, Y. Robert. A proposal for a heterogeneous cluster ScaLAPACK (dense linear solvers), IEEE Trans. Computers, 50 (2001), 1052-1070.

[6] O. Beaumont, V. Boudet, F. Rastello, Y. Robert, Matrix-matrix multiplication on heterogeneous platforms, Technical Report RR-2000-02, LIP, ENS Lyon (2000).

[7] O. Beaumont, V. Boudet, F. Rastello, Y. Robert, Matrix multiplication on heterogeneous platforms, IEEE Transactions on Parallel and Distributed Systems, 12 (2001), 1033-1051.

[8] O. Beaumont, A. Legrand, Y. Robert, The master-slave paradigm with heterogeneous processors, Technical Report RR-2001-13, LIP, ENS Lyon (2001).

[9] O. Beaumont, A. Legrand, F. Rastello, Y. Robert, Dense linear algebra kernels on heterogeneous platforms: redistribution issue, Parallel Computing, 28 (2002), 155-185.

[10] S.W. Bowa et al, Dual-Level Parallel Analysis of Harbor Wave Response Using MPI and OpenMP Source, International Journal of High Performance Computing Applications, 14 (2000), 49-64.

[11] R. Brightwell, L.A. Fisk, D.S. Greenberg, T. Hudson, M. Levenhagen, A.B. Maccabe, R. Riesen, Massively parallel computing using commodity components, Parallel Computing, 26 (2000), 243266.

[12] L.E. Cannon, A cellular computer to implement the Kalman filter algorithm, Ph.D. Thesis, Montana State University, Bozman, Montana. 
[13] J. Choi, J. Dongarra, R. Pozo, D.W. Walker, ScaLAPACK: A scalable linear algebra library for distributed memory concurrent computers, In: Proceedings of the Fourth Symposium on the Frontiers of Massively Parallel Computation, McLean, Virginia, October 19-21, (1992), 120-127.

[14] J. Choi, J. Dongarra, D.W. Walker, The design of scalable software libraries for distributed memory concurrent computers, in J. Dongarra, B. Tourancheau (Eds.), Environments and Tools for Parallel Scientific Computing, Elsevier, Amsterdam (1992).

[15] J. Dean, S. Ghemawat, MapReduce: Simplified Data Processing on Large Clusters, In: Proceedings of the Sixth Symposium on Operating System Design and Implementation, San Francisco, CA, December, (2004).

[16] J. Dongarra, C.B. Moler, J.R. Bunch, G.W. Stewart, LINPACK User's Guide, SIAM, Philadelphia (1979).

[17] J. Dongarra, R.C. Whaley, A User's Guide to the BLACS v1.1 (1997). http://netlib.org/blacs/lawn94.ps

[18] J. Dongarra, J.F. Pineau, Y. Robert, F. Vivien, Matrix Product on Heterogeneous Master-Worker Platforms, PPoPP 2008, (2008).

[19] G. Fox, M. Johnson, G. Lyzenga, S. Otto, J. Salmon, D. Walker, Solving Problems on Concurrent Processors, v. I, Prentice-Hall, Englewood Cliffs, NJ (1988).

[20] R. van de Geijn, J. Watts, SUMMA: Scalable Universal Matrix Multiplication Algorithm, Concurrency: Practice and Experience, 9 (1997), 255-274.

[21] A. Gramma, A. Gupta, G. Karypis, V. Kumar, Introduction to parallel computing, Addison-Wesley (2003).

[22] V. Grolmusz, Co-Orthogonal Codes. Designs, Codes and Cryptography, 38 (2006), 363-372.

[23] Y. He, H.Q.C. Ding, Using Accurate Arithmetics to Improve Numerical Reproducibility and Stability in Parallel Applications, In: Proceedings of the 14th International Conference on Supercomputing, (2000), 225 - 234.

[24] I. Ioannidis, A. Grama, M. Atallah, A secure protocol for computing dotproducts in clustered and distributed environments, In: Proceedings of International Conference on Parallel Processing 2002, IEEE Computer Society, (2002), 379 - 384 . 
[25] A. Kalinov, Scalability analysis of matrix-matrix multiplication on heterogeneous clusters, In: Proceedings of ISPDC 2004 - HeteroPar 04, IEEE Computer Society (2004).

[26] A. Kalinov, A. Lastovetsky, Y. Robert, Heterogeneous computing, Parallel computing, 31 (2005), 649652.

[27] A. Kalinov, A. Lastovetsky, Heterogeneous distribution of computations solving linear algebra problems on networks of heterogeneous computers, Journal of Parallel and Distributed Computing, 61 (2001), 520-535.

[28] A. Lastovetsky, Parallel Computing on Heterogeneous Networks, WileyInterscience, Hoboken, NJ, 2003.

[29] A. Lastovetsky, R. Reddy, On performance analysis of heterogeneous algorithms, Parallel Computing, 30 (2004), 11951216.

[30] K. Li, Fast and Scalable parallel matrix computations on distributed memory systems, In: Proceedings of the 19th IEEE International Parallel and Distributed Processing Symposium (IPDPS'05), (2005), 307-314.

[31] K. Li, V. Y. Pan. Parallel matrix multiplication on a linear array with a reconfigurable pipelined bus system, IEEE Trans. on Computers, 50 (2001), 519-525.

[32] E.B. Lum, K.L. Ma, Hardware-Accelerated Parallel Non-Photorealistic Volume Rendering, In: Proceedings of the 2nd International Symposium on Non-photorealistic Animation and Rendering (2002), 66.

[33] P.D. Michailidis, T. Typou, V. Stefanidis, K.G. Margaritis, Performance Models for Matrix Computations on Networks of Heterogeneous Workstations, Neural, Parallel and Scientific Computations, 14 (2006), 177 - 204.

[34] Y. Ohtaki, D. Takahashi, T. Boku, M. Sato, Parallel implementation of Srassen's matrix multiplication algorithm for heterogeneous clusters, In: Proceedings of 18th International Parallel and Distributed Processing Symposium (IPDPS 2004), IEEE Computer Society, CD-ROM (2004).

[35] P. Pacheco, Parallel programming with MPI, San Francisco, CA, Morgan Kaufmann (1997).

[36] P.M. Papadopoulos, M.J. Katz, G. Bruno, NPACI Rocks: Tools and techniques for easily deploying manageable Linux clusters, San Siego Supercomputer Center, University of California San Siego (2001). 
[37] J. Peinado, A.M. Vidal, Several Parallel Algorithms for Solving Nonlinear Systems with Symmetric and Positive Definite Jacobians, In: Proceedings of the International Parallel and Distributed Processing Symposium (IPDPS '03), IEEE Computer Society (2003).

[38] J. Peinado, A.M. Vidal, Three Parallel Algorithms for Solving Nonlinear Systems and Optimization Problems, In: Proceedings of VECPAR 05, Springer-Verlag (2005), 657-670.

[39] M. Snir, S. Otto, S. Huss-Lederman, D.W. Walker, J. Dongarra, MPI: The Complete Reference, The MIT Press, Cambridge, Massachusetts (1996).

[40] F. Tinetti, A. Quijano, A. Giusti, E. Luque, Heterogeneous networks of workstations and the parallel matrix multiplication, In: Proceedings of the Euro PVM/MPI 2001, Springer-Verlag, (2001), 296- 303.

[41] L. Zhuo, V. K. Prasanna. Scalable and modular algorithms for floatingpoint matrix multiplication on reconfigurable computing systems, IEEE Trans. on Parallel and Distributed Systems, 18 (2007), 433-448.

[42] Y. Yan, X. Zhang, Y. Song, An effective and practical performance prediction model for parallel computing on non-dedicated heterogeneous NOW, Journal of Parallel and Distributed Computing, 38 (1996), 63-80.

[43] B. Wilkinson, M. Allen, Parallel programming: Techniques and applications using networking workstations, Prentice-Hall, Inc (2005).

[44] http://hadoop.apache.org/ 
